\title{
Left Inferior Frontal Activations Differentially Modulated by Scrambling in Ditransitive Sentences
}

\author{
Masatoshi Koizumi ${ }^{*}$, , Jungho Kim ${ }^{1}$, Naoki Kimura ${ }^{1}$, Satoru Yokoyama ${ }^{2}$, Shigeru Sato ${ }^{3}$, \\ Kaoru Horie $^{4}$ and Ryuta Kawashima ${ }^{2}$ \\ ${ }^{I}$ Graduate School of Arts and Letters, Tohoku University, Sendai, Japan \\ ${ }^{2}$ Institute of Development, Aging and Cancer, Tohoku University, Sendai, Japan \\ ${ }^{3}$ Sendai University, Shibata, Japan \\ ${ }^{4}$ Graduate School of Languages and Cultures, Nagoya University, Nagoya, Japan
}

\begin{abstract}
In order to clarify the relationship among grammatical knowledge, processing components, and neural substrates in sentence comprehension, we used functional magnetic resonance imaging to investigate how brain activation is affected by two types of scrambling (short scrambling and middle scrambling) in ditransitive sentences in Japanese. Short scrambling and middle scrambling enhanced activation in the anterior and posterior left inferior frontal gyrus respectively. This finding accords with the view that the anterior left inferior frontal gyrus is involved in the automatic processing that establishes a dependency relation between a verb and its arguments, and the posterior left inferior frontal gyrus supports this kind of processing through its role in verbal working memory. This result is more congruent with a process-based approach to neural bases for sentence processing, which searches for neurological correlates of psycholinguistically defined processing components, than with a grammar-based approach, which probes neural networks with the assumption that major grammatical operations are neurologically individuated.
\end{abstract}

Keywords: Functional magnetic resonance imaging, Japanese, scrambling, sentence processing, syntax, verbal working memory.

\section{INTRODUCTION}

Functional magnetic resonance imaging (fMRI) studies on sentence processing have revealed that cortical activations in the left inferior frontal gyrus (IFG) are differentially modulated by various factors such as syntactic complexity and verbal working memory load, as well as hierarchies of thematic roles, case, and animacy of nouns [1-13]. In particular, activation in the posterior left IFG (pLIFG: BA 44/13) is sensitive to verbal working memory demands, a phenomenon that suggests the involve-ment of the pLIFG in working memory processes $[8,14]$. In contrast, activation in the anterior left IFG (aLIFG: BA 45/46) is enhanced more by grammatical factors than memory load, thus implicating the aLIFG in a subcomponent of syntactic processing such as establishing a dependency relation between a verb and its arguments $[15,16]$. Functional distinctions between the pLIFG and aLIFG have been investigated in lesion studies as well $[17,18]$. For example, [18] found that patients with lesions in the pars opercularis (pLIFG) showed greater difficulty in comprehending syntactically demanding sentences, whereas a patient with a lesion in the pars triangularis (aLIFG) misinterpreted sentences with a noncanonical word order more frequently; these results are congruent with the results of imaging studies. Note that the role sharing between the pLIFG and aLIFG has physiological bases: the pLIFG is

*Address correspondence to this author at the Graduate School of Arts and Letters, Tohoku University, 27-1 Kawauchi, Aoba-ku, Sendai, 980-8576, Japan; Tel: +81-22-795-5981; Fax: +81-22-795-5981;

E-mail:koizumi@sal.tohoku.ac.jp part of a dorsal pathway connected to the posterior superior temporal gyrus via the inferior parietal lobule, whereas the aLIFG is more strongly linked to the temporal pole and posterior mid temporal gyrus through a ventral pathway $[19$, $20]$.

In accordance with these general findings, scrambling in Japanese yields higher activation in the aLIFG [21, 22]. Most imaging studies of scrambling in Japanese, as well as in other free word order languages such as German, have focused on a single type of scrambling - the scrambling of the object $(\mathrm{O})$ of a (mono-)transitive verb across the subject (S), as illustrated by the Japanese sentences in (1). (1a) is a canonically ordered transitive sentence (SO), and (1b) is its scrambled version with a syntactic movement of the accusative direct object across the nominative subject (OS) [23, 24]. This type of syntactic movement is sometimes called middle scrambling or M-scrambling ([25]; see also [26]). Verbs generally appear at the end of sentences in Japanese (see Fig. 1A). Increased activation in the aLIFG has been reported for sentences with the OS word order compared with those with the SO word order.

$\begin{array}{lll}\text { a. Taro-ga Hanako-o hometa. } & \text { Taro-NOM Hanako-ACC } & \text { praised } \\ & \text { 'Taro praised Hanako.' } & \\ \text { b. } & \text { Hanako-o } \quad \text { Taro-ga hometa. } \\ & \text { Hanako-ACC Taro-NOM praised } \\ & & \end{array}$

[SO] 
A.

$$
\begin{array}{ll}
\text { SO: } & {[\text { NP-NOM [NP-ACC V]] }} \\
\text { OS: } & {\left[\text { NP-ACC [NP-NOM } \left[\_\right.\right. \text {V]]] }} \\
& \quad \text { M-scrambling }
\end{array}
$$

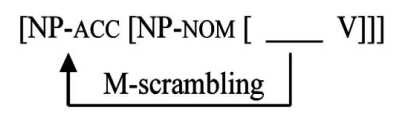

B.

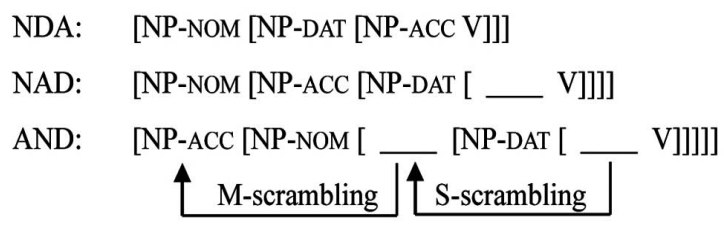

Fig. (1). Illustration of short-scrambling and middle-scrambling in (mono-)transitive sentences (A) and ditransitive sentences (B).

However, the influence of other types of scrambling on cortical activations during sentence processing has not yet been clarified. To fill the gap, in this paper we report the results of a study dealing with two types of scrambling involved in ditransitive sentences in Japanese. The canonical word order of Japanese ditransitive sentences is NDA and is shown in (2a): the nominative subject $(\mathrm{N})$ is followed by the dative indirect object (D) and accusative direct object (A) $[25,27,28]$. (2b) shows the scrambling of the accusative direct object across the dative indirect object (NAD). This type of scrambling is called short scrambling or S-scrambling. In (2c), the accusative direct object is dislocated to the left of the subject (AND). According to current linguistic analyses [25, 26, 29], (2c) involves two instances of scrambling: (i) scrambling of the direct object across the indirect object, that is, S-scrambling and (ii) scrambling of the already scrambled direct object across the subject, that is, Mscrambling (see Fig. 1B).

(2)

$$
\begin{aligned}
& \text { a. Taro-ga Hanako-ni ringo-o ageta } \\
& \text { Taro-NOM Hanako-DAT apple-ACC gave } \\
& \text { 'Taro gave an apple to Hanako. } \\
& \text { b. Taro-ga ringo-o Hanako-ni ageta. } \\
& \text { Taro-NOM apple-ACC Hanako-DAT gave } \\
& \text { c. Ringo-o Taro-ga Hanako-ni ageta. } \\
& \text { apple-ACC Taro-NOM Hanako-DAT gave }
\end{aligned}
$$

[NDA]

[NAD]

[AND]

S-scrambling and M-scrambling have different grammatical properties. Putting aside technical details, we note that S-scrambling is similar to the movement of the underlying object to the surface subject position in English passive sentences (e.g., The city was destroyed _ by the enemy in 1564). M-scrambling shares grammatical properties with wh-movement (e.g., What did you buy _?) as well as with passive movement in English. Although several previous studies on scrambling in German and Japanese involving event-related brain potentials reported anterior negativity and/or P600-like components for various instances of scrambling [30-34], no functional imaging study has examined Sscrambling and $\mathrm{M}$-scrambling in ditransitive sentences.

By directly comparing ditransitive sentences that have the above three word orders, we investigated how S-scrambling and M-scrambling in ditransitive sentences would modulate cortical activations so that we could clarify the relationship among grammatical knowledge, processing components, and neural substrates in sentence comprehension. We tested the following three hypotheses: First, given that NDA is canonical and NAD and AND involve one and two instances of scrambling respectively, the three conditions are associated with a parametric increase in linguistic complexity. Cortical activations therefore could be enhanced in this particular order (Hypothesis 1). This is a quantitative hypothesis. On the other hand, S-scrambling and Mscrambling may have different qualitative effects on cortical activations. There are two major approaches to study such qualitative differences-a grammar-based approach and a process-based one [35].

The grammar-based approach probes into the neural bases of the human language faculty with the methodological assumption that major grammatical operations are neurologically individuated. This approach would thus predict that (i) NAD and AND, when compared with NDA, activate the same cortical regions because they both involve the same type of movement operation grammatically, that is, Sscrambling and (ii) AND is associated with an additional activated region (possibly in the aLIFG), as it includes an instance of M-scrambling that is grammatically distinct from $\mathrm{S}$-scrambling but is parallel to the M-scrambling in transitive sentences such as (1b) (Hypothesis 2).

The process-based approach, on the other hand, assumes that psycholinguistically defined processing components are mapped onto brain space and time. According to this approach, NAD vs. NDA should activate the same region as OS vs. SO (i.e., the aLIFG), because the processing of NAD as well as that of OS requires the integration of a filler into a gap position, thereby establishing the dependency between the scrambled item and its original position, as indicated by an underline in Fig. (1) (first half of Hypothesis 3). The processing of AND also involves the integration of a filler into a gap position, but the distance between the scrambled object to the left of the subject and its original position adjacent to the verb in AND is greater than the distance between the object and its original position in NAD. AND therefore requires the maintenance of the filler in working memory until the gap is encountered, thus imposing additional costs on verbal working memory, which in turn may yield higher activations in the pLIFG (second half of Hypothesis 3 ). Hypothesis 1 may be compatible with either Hypotheses 2 or 3 , but the last two hypotheses are mutually exclusive.

\section{MATERIALS AND METHODOLOGY}

\subsection{Participants}

47 healthy native speakers of Japanese (33 men and 14 women) participated in the experiment. Their age ranged from 18 to 30 years (mean age $=22.1, \mathrm{SD}=2.9$ ). All the participants were right-handed. The handedness survey was based on the Edinburgh Handedness Inventory [36]. Before the experiment, all participants were provided with a sufficient explanation of the entire experiment and its safeness, in accordance with the guidelines of Tohoku University. Only those participants who gave written informed consent took part in the actual experiment. 


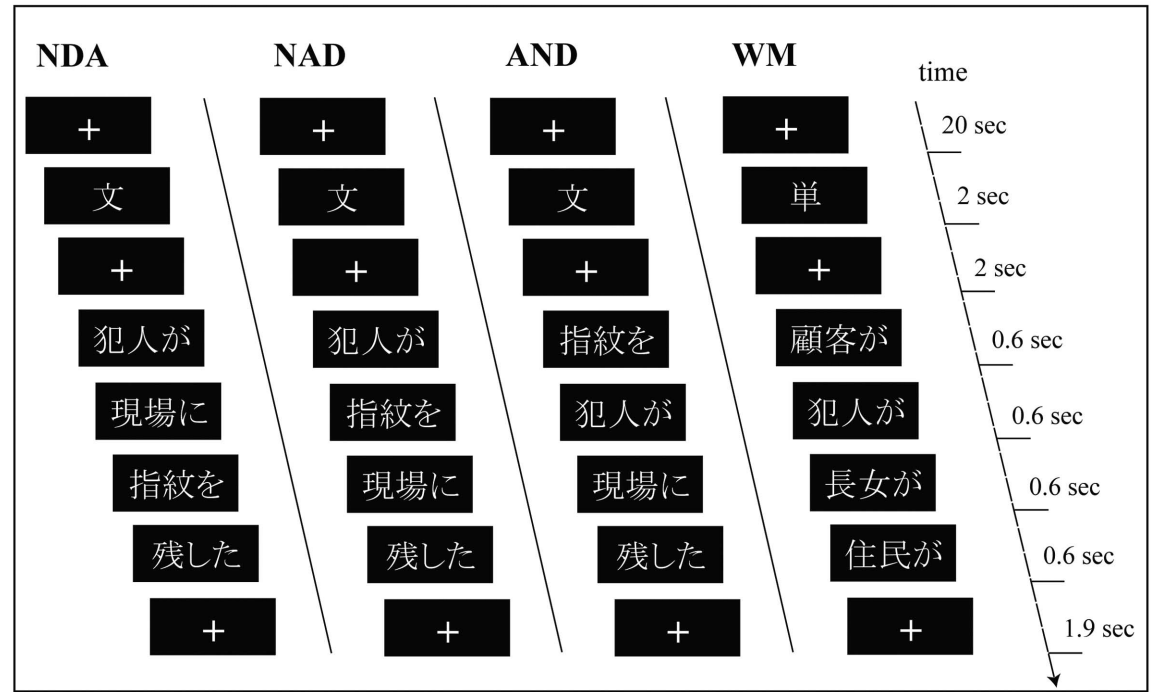

Fig. (2). Presentation method for task conditions. Each phrase was shown at the center of the screen for $600 \mathrm{~ms}$, with a fixation cross at the beginning and end of each sentence.

\subsection{Materials and Design}

Following Caplan et al.'s previous studies, we conducted an fMRI experiment with the sentence plausibility judgment task $[7,8]$. The participants were asked to determine the plausibility of ditransitive sentences with three different word orders. The total number of stimuli (144) was divided across two sessions with 72 stimuli each. The two sessions consisted of five conditions: nominative-dative-accusative order (NDA), nominative-accusative-dative order (NAD), accusative-nominative-dative order (AND), working memory (WM) condition, and rest (R) condition. The 144 stimuli were equally distributed across the four task conditions (NDA, NAD, AND, and WM); that is, each condition had 36 stimuli. In each of the three sentence conditions (NDA, NAD, and AND), there were 24 semantically plausible ("correct") ditransitive sentences and 12 semantically implausible ("incorrect") ditransitive sentences. We used these semantically implausible sentences to ensure that participants did not digress from the task. To avoid the possibility of participants making semantic judgments before encountering the verb, we prepared the stimuli in such a way that semantic anomalies could only be detected at the point of the verb. The implausible sentences were constructed in violation of the congruity between the verb and one of the three arguments. For example, in Tomoko-ga Taroo-ni yakusoku-o modosita "Tomoko returned a promise to Taro," the verb modosita "returned" and the direct object yakusoku "promise" are thematically incompatible. The sentence will be plausible if either the verb or the direct object is replaced with an appropriate verb or noun, as in Tomoko-ga Taroo-ni yakusoku-o tutaeta "Tomoko conveyed a promise to Taro" for instance. As the three conditions (i.e., NAD, NAD, and AND) are all comparable in terms of semantic (im)plausibility, the effect of semantic (im)plausibility to cortical activation, if any, should be cancelled out when these conditions are directly compared. The WM condition contained 36 sequences of words. Each sequence in the WM condition contained four nouns with unified case particles or four verbs in the past tense. The stimuli in the WM condition were prepared from the nouns and verbs that were used for the sentence conditions; they were prepared in such a way that they could not be construed as complete sentences. A stimulus with four different words was treated as a "correct" stimulus, whereas a stimulus that had a pair of the same words was regarded as an "incorrect" stimulus. We used 24 correct stimuli and 12 incorrect stimuli (Fig. 2).

The experiment was based on block design: Each block contained six sentences or six word lists and was repeated thrice in a session (and hence six times in the entire experiment). At the beginning of each block, either the Japanese word bun "sentence" or tango "word" was shown to indicate the type of the block. The blocks were presented on the screen randomly, while the order of the stimuli in each block remained constant (pseudo-randomized) (Fig. 3). The duration of a block was $25,800 \mathrm{~ms}$, with $24,000 \mathrm{~ms}$ of $\mathrm{R}$ condition between task blocks. In the task blocks, the stimuli were presented in an automatic phrase-by-phrase manner; each of them took $4,300 \mathrm{~ms}$. In the three sentence conditions, participants were asked to judge whether the sentences they saw on the screen were semantically plausible. In the WM condition, the participants were instructed to judge whether the lists of four words included a pair of the same words. The response time for each stimulus was recorded between the onset of the fourth word and the pressing of the button.

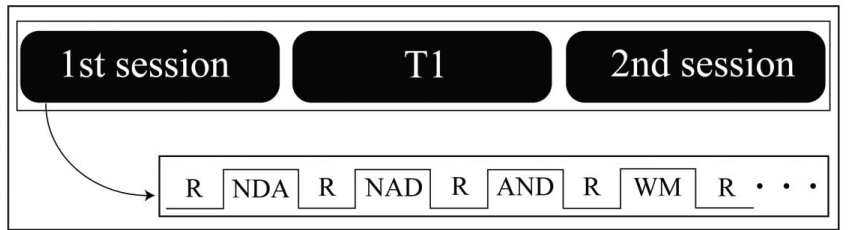

Fig. (3). Design of sequential blocks in the current study. Task blocks were randomly presented between rest $(\mathrm{R})$ conditions.

\section{3. fMRI Measurement}

The image scans were acquired with a 1.5 Tesla Siemens system using an echo-planar imaging (EPI) sequence $(\mathrm{TR}=$ $3000 \mathrm{~ms}, \mathrm{TE}=50 \mathrm{~ms}$, acquisition matrix $=64 \times 64, \mathrm{FOV}=$ $192 \mathrm{~mm}$, slices $=42$, slice thickness $=3 \mathrm{~mm}$ ). Between the two sessions, anatomical T1-weighted MDEFT images (thickness $=1 \mathrm{~mm}, \mathrm{FOV}=256 \mathrm{~mm}$, data matrix $=192 \times$ $224, \mathrm{TR}=1900 \mathrm{~ms}, \mathrm{TE}=3.93 \mathrm{~ms}$ ) were acquired from all 
participants. Each session lasted 10 minutes and 48.8 seconds, and the entire experiment took about 30 minutes for each participant.

\section{4. fMRI Data Analysis}

To specify the cortical region exhibiting activation during the various tasks, we carried out statistical analyses of image processing by using SPM2, which was developed by the Wellcome Department of Cognitive Neurology and operates on a MATLAB platform. The origin of EPI and T1-weighted anatomical images were synchronized to align the head position. The brain forms acquired from each participant were anatomically normalized to the Montreal Neurological Institute (MNI) standard brain template, and images were smoothed using a $9 \mathrm{~mm}$ Gaussian filter. Statistical analyses of the tasks for each participant and the entire group were conducted at the first and second stages, respectively. We set the statistical threshold at $p<0.05$ (corrected for family-wise error (FWE) rate). Finally, we performed region of interest (ROI) analyses in brain areas obtained from the [NAD vs. NDA] and [AND vs. NAD] comparisons.

\section{RESULTS}

\subsection{Behavioral Data}

The reaction time of two participants could not be recorded owing to a failure in the button board. Thus, our final ANOVA analysis included the data of 45 out of the 47 participants. Reaction time (RT) and error rate (ER) are summarized in Table 1. A comparison of RTs and ERs across all the task conditions did not show any significant difference $[\mathrm{RT}: F(3,132)=0.27, p=.84 ; \mathrm{ER}: F(3,132)=.78$, $p=.51]$.

Table 1. Mean (M) and Standard Deviation (SD) for Reaction Time (RT) in Milliseconds, and Error Rate (ER) for Each Condition

\begin{tabular}{|c|c|c|c|c|}
\hline \multirow{2}{*}{} & \multicolumn{2}{|c|}{ Reaction Time } & \multicolumn{2}{c|}{ Error Rate } \\
\cline { 2 - 5 } & Mean (ms) & SD & Mean (\%) & SD \\
\hline \hline NDA & 949.9 & 167.7 & 7.7 & 8.2 \\
\hline NAD & 942.7 & 188.8 & 6.7 & 9.0 \\
\hline AND & 953.9 & 185.2 & 6.9 & 6.9 \\
\hline WM & 944.8 & 151.6 & 6.3 & 9.7 \\
\hline & \multicolumn{2}{|c|}{$F(3,132)=0.27, p=0.84$} & $F(3,132)=0.78, p=0.51$ \\
\hline
\end{tabular}

\subsection{Imaging Data}

The activated brain regions in the comparison of wordorder variations and R/WM conditions are shown in Fig. (4) and Table 2 . A comparison of the task conditions with the $\mathrm{R}$ condition showed activations in the bilateral inferior frontal gyrus (IFG), bilateral middle frontal gyrus (MFG), and left middle and superior temporal gyri (LSTG and LMTG). On the other hand, the comparison of the task conditions and the WM condition revealed activations in the left inferior frontal gyrus (LIFG) and left middle and superior temporal gyri (LSTG and LMTG).

Finally, the direct comparisons of the task conditions are shown in Fig. (5) and Table 3. All comparisons involving the cortical specification of scrambling showed activation in LIFG.

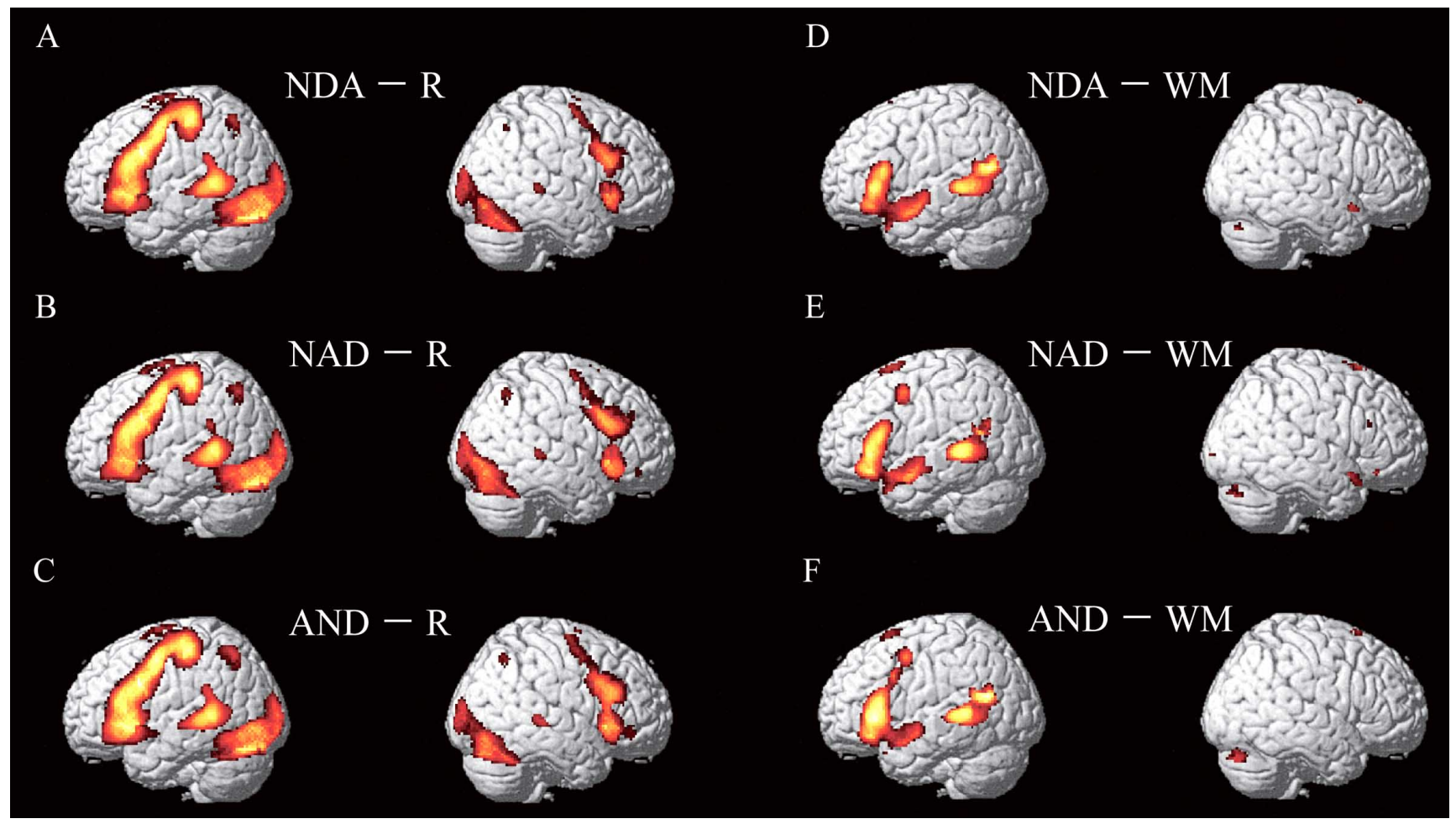

Fig. (4). $\mathrm{R}$ condition and WM condition subtracted from the three sentence types of the task condition. Task condition $v s$. R condition shows the regions of activation that are inherent to the processing of each task $(\mathbf{A}, \mathbf{B}$, and $\mathbf{C})$, while task condition $v s$. WM condition shows the activation of grammatical processing apart from lexical effects in the stimuli. 
Table 2. Activated Brain Regions and $t$ Values

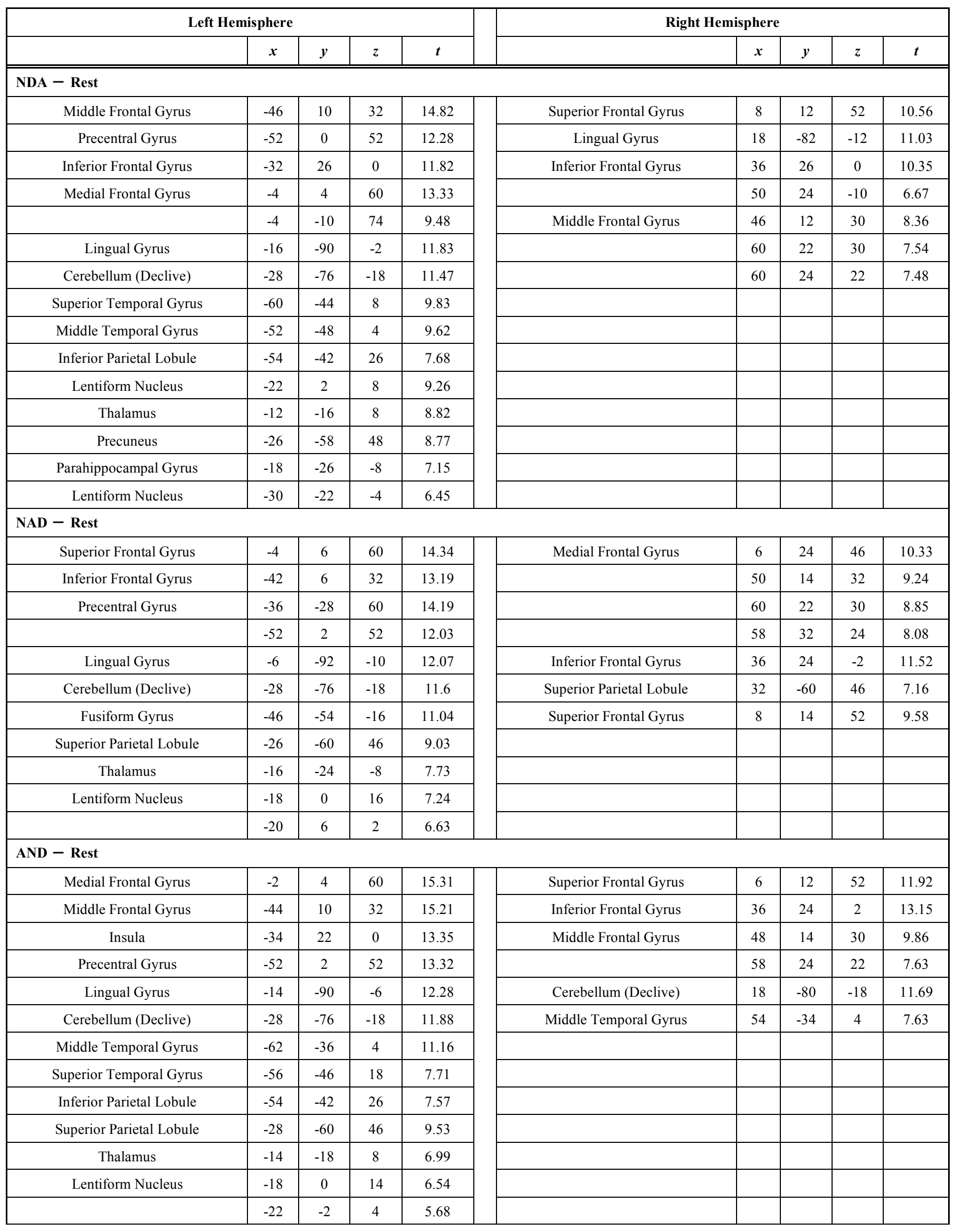


(Table 2) Contd.....

\begin{tabular}{|c|c|c|c|c|c|c|c|c|c|}
\hline \multicolumn{5}{|c|}{ Left Hemisphere } & \multicolumn{5}{|c|}{ Right Hemisphere } \\
\hline \multicolumn{10}{|l|}{ NDA - WM } \\
\hline \multirow[t]{2}{*}{ Inferior Frontal Gyrus } & -52 & 28 & 4 & 10.57 & Cerebellum (Tuber) & 26 & -80 & -30 & 7.41 \\
\hline & -56 & 24 & 14 & 9.51 & & & & & \\
\hline Middle Temporal Gyrus & -66 & -54 & 4 & 7.04 & & & & & \\
\hline Posterior Cingulate & -2 & -56 & 6 & 9.92 & & & & & \\
\hline \multirow[t]{2}{*}{ Cerebellum (Culmen) } & -6 & -44 & 2 & 5.8 & & & & & \\
\hline & -28 & -32 & -20 & 8.52 & & & & & \\
\hline Parahippocampal Gyrus & -26 & -14 & -14 & 6.27 & & & & & \\
\hline \multicolumn{10}{|l|}{ NAD - WM } \\
\hline \multirow[t]{2}{*}{ Middle Frontal Gyrus } & -42 & 34 & -14 & 10.18 & Cerebellum (Pyramis) & 22 & -78 & -32 & 8.35 \\
\hline & -42 & 4 & 46 & 7.82 & Parahippocampal Gyrus & 24 & -12 & -16 & 6.69 \\
\hline \multirow[t]{3}{*}{ Middle Temporal Gyrus } & -58 & 4 & -20 & 10.15 & & & & & \\
\hline & -64 & -42 & 2 & 9.19 & & & & & \\
\hline & -42 & -62 & 24 & 8.02 & & & & & \\
\hline Inferior Frontal Gyrus & -52 & 26 & 12 & 10.12 & & & & & \\
\hline \multirow[t]{3}{*}{ Middle Temporal Gyrus } & -46 & -66 & 20 & 9.57 & & 16 & -80 & -32 & 8.6 \\
\hline & -64 & -58 & 4 & 7.18 & Cerebellum (Uvula) & 16 & -78 & -24 & 7.36 \\
\hline & -56 & 6 & -20 & 8.22 & & & & & \\
\hline \multirow[t]{2}{*}{ Inferior Frontal Gyrus } & -50 & 28 & 4 & 9.61 & & & & & \\
\hline & -52 & 26 & 14 & 9.2 & & & & & \\
\hline Superior Frontal Gyrus & -6 & 16 & 64 & 6.17 & & & & & \\
\hline
\end{tabular}

The data clearly show that sentences that involve scrambling activate regions in the LIFG. When the NDA condition was subtracted from the NAD condition, significant activation was observed in the aLIFG $(-42,32,30)$, whereas subtracting NAD from NDA showed no activation in the relevant brain region. This finding confirms our initial assumption that the canonical word order of Japanese ditransitive sentences is NDA [18, 22, 38]. When NDA was subtracted from AND, a larger activated area was revealed; this area extended from the aLIFG to pLIFG. The comparison of AND $v s$. NAD revealed an activated area in the pLIFG (-38, $20,6)$. Crucially, however, no regions in LIFG (or any other part of the brain) showed a parametric sensitivity to a sentence's structural complexity, with enhanced activation in the order of NDA $<$ NAD $<$ AND. Therefore, Hypothesis 1 was not supported. The region revealed by the comparison of NAD vs. NDA was in the aLIFG, and its activation peak was close to what was reported in previous studies on scrambling involved in Japanese transitive sentences (i.e., M-scrambling) such as (1b) $[19,21]$. The same region was also activated in AND when compared with NDA. In contrast, AND vs. NAD yielded enhanced activation in a distinct region in the pLIFG. Thus, Hypothesis 3, that is, (OS vs. SO) $=($ NAD vs. $\mathrm{NDA}) \neq(\mathrm{AND} v s$. NAD) is supported, but Hypothesis 2, that 


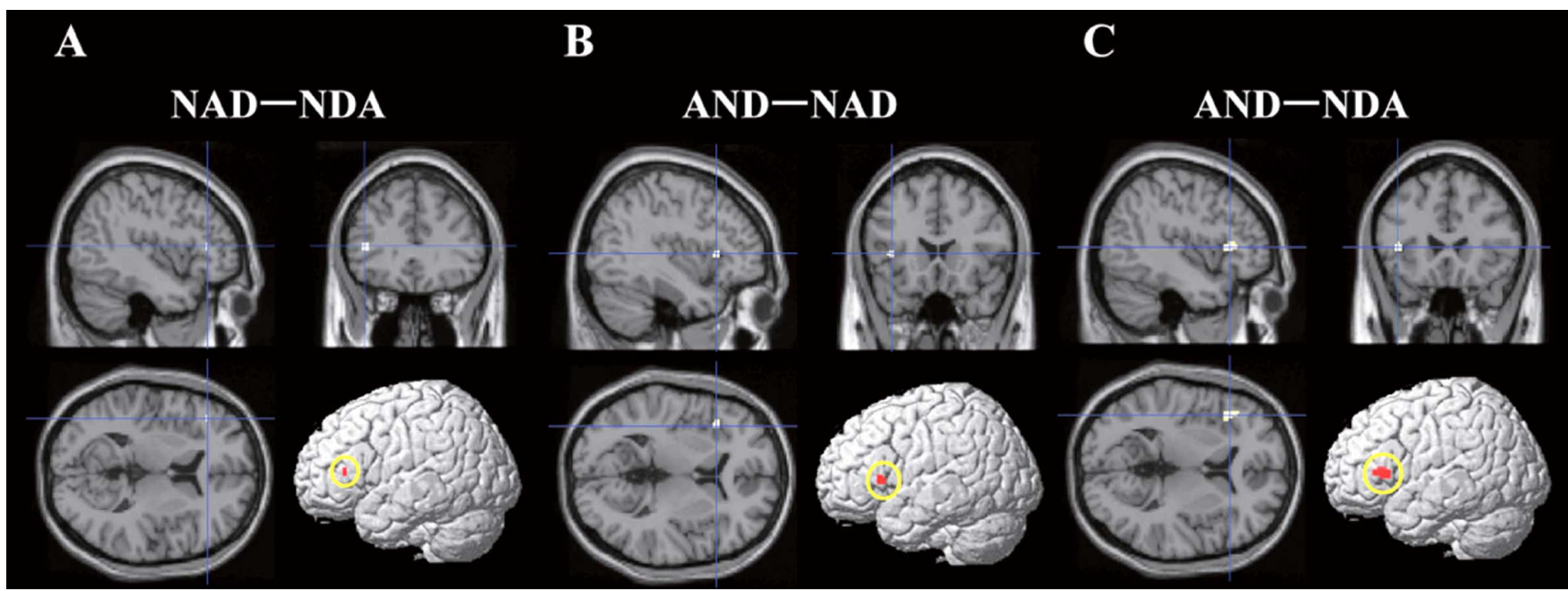

Fig. (5). Comparisons of brain activation across different conditions. NAD vs. NDA shows activation in the dorsal direction of Broca's area, while AND vs. NAD shows activation in the ventral section.

Table 3. Activated Brain Regions in Crucial Contrasts

\begin{tabular}{|c|c|c|c|c|}
\hline \multicolumn{5}{|c|}{ Left Hemisphere } \\
\hline & $\boldsymbol{x}$ & $\boldsymbol{y}$ & $\boldsymbol{z}$ & $\boldsymbol{t}$ \\
\hline \hline NAD - NDA & -42 & 32 & 10 & 5.29 \\
\hline Inferior Frontal Gyrus & -38 & 20 & 6 & 5.5 \\
\hline AND - NAD & & \\
\hline Inferior Frontal Gyrus & -42 & 24 & 6 & 5.7 \\
\hline AND - NDA
\end{tabular}

is, $(\mathrm{OS} v s . \mathrm{SO})=(\mathrm{AND} v s . \mathrm{NAD}) \neq(\mathrm{NAD} v s$. NDA) is not. The WM condition, when compared with the $\mathrm{R}$ condition, activated regions in the pLIFG but failed to do so in the aLIFG. All these points are illustrated by the region of interest (ROI) analyses (Fig. 6).

\section{DISCUSSION}

\subsection{Three Hypotheses}

The present study aimed to test three hypotheses: Hypothesis 1 (given that the three conditions (NDA, NAD, and AND) are associated with a parametric increase of linguistic complexity, cortical activations will be enhanced in this particular order); Hypothesis 2 (NAD and AND, when compared with NDA, activate the same cortical regions because they both involve S-scrambling, and AND is associated with an additional activated region (i.e., the aLIFG), as it includes an instance of M-scrambling, which is grammatically distinct from S-scrambling); Hypothesis 3 (NAD $v s$. NDA activates the same region as OS vs. SO (i.e., the aLIFG), because the processing of NAD as well as that of OS requires the integration of a filler into a gap position, whereas AND may yield additional activations in the pLIFG because it requires the filler to be maintained in working memory until the gap is encountered). Hypotheses 2 and 3 are based on the grammar- and process-based approaches, respectively [35]. The overall results suggest that the aLIFG is involved in constructing filler-gap dependencies (i.e., integration) regardless of the grammatical type (S-scrambling/ M-scrambling), whereas the pLIFG makes a larger contribution when a longer dependency is processed, possibly owing to its role in verbal working memory (i.e., maintenance). No brain regions showed a parametric sensitivity to the number of movements involved. These results are congruent with the predictions by Hypothesis 2 but not with those by Hypotheses 1 or 3 . The process-based approach is therefore supported. This result suggests that psycholinguistically defined processing components such as integration and maintenance, as opposed to grammatical distinctions such as S- vs. Mscrambling, are mapped onto brain space and time.

Looking at the ROI analyses shown in Fig. (6), one might wonder if the aLIFG supports S-scrambling whereas the pLIFG supports M-scrambling. Note, however, that the Mscrambling involved in Japanese (mono-)transitive sentences such as (1b) yields enhanced activation in the aLIFG rather than in the pLIFG. It is thus safe to conclude that the division of functional roles between the aLIFG and pLIFG does not quite parallel the grammatical distinction between S-scrambling and M-scrambling.

Our conclusion is consistent with the findings regarding filler-gap dependencies in languages other than Japanese that were previously mentioned in the introduction section. [14], for instance, has reported enhanced activation both in the aLIFG and pLIFG for sentences with an object relative clause, which contains a long filler-gap dependency (e.g., The money that the robber stole was in the bank vault.), as compared with sentences with a subject relative clause, which contains a shorter dependency (e.g., The robber that stole the money was in the bank vault.). During concurrent speech articulation, however, this complexity effect was eliminated in the pLIFG but not in the aLIFG, indicating that the pLIFG supports sentence comprehension through its role in articulatory rehearsal whereas the aLIFG is concerned more with combinatorial processes.

A study on German scrambling [37] has observed a parametric sensitivity of LIFG activation to the number of 


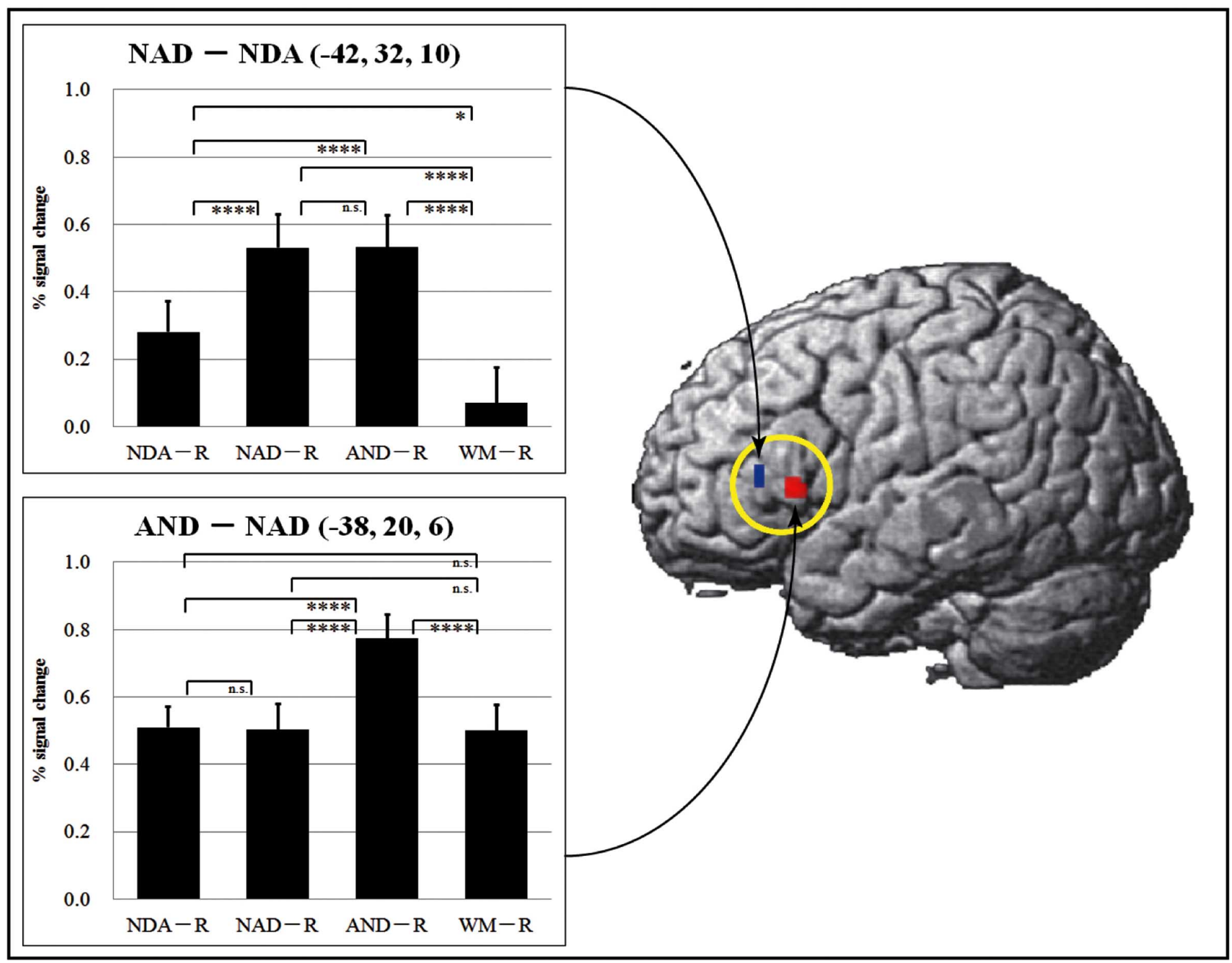

Fig. (6). Region-of-interest (ROI) analysis of the activated areas in the current experiment. The activated brain region for NAD vs. NDA (short-scrambling) is shown in blue color, while AND vs. NAD (middle-scrambling) is shown in red.

scramblings. The study compared German ditransitive sentences with three different word orders: (i) sentences with the canonical order, (ii) sentences in which the indirect object is fronted across the subject, and (iii) sentences where both the direct and indirect objects have been scrambled across the subject. They found that (i) resulted in the least activation in the Broca's area, followed by (ii) and (iii); the activation is correlated with the number of movements involved. This might appear to contradict the present study's result that observed no parametric modulation of the cortical activation. Note, however, that what differs among the three conditions in [37] is the number of noun phrases that are moved: zero, one, and two noun phrases are moved in (i), (ii), and (iii), respectively. In contrast, what differs among the three conditions in the present study is the distance between the filler and the gap: zero, one noun, and two nouns in NDA, NAD, and AND, respectively. Crucially, both in NAD and AND, only one noun phrase, the accusative direct object, is moved. This means that the condition (iii) sentences in [37] are much more complex than the AND sentences in the present study in terms of their syntactic structures as well as in terms of processing components required to comprehend them. This may be the reason for the partially different experimental results between the two studies.

\section{2. aLIFG vs. pLIFG}

The aLIFG activation during the comprehension of NAD when compared with NDA may reflect the unconscious, automatic processing for simple syntactic operations such as the construction of a short filler-gap dependency. The pLIFG activation, on the other hand, may reflect more intentional, deliberate processing that requires rather conscious processing. Since the AND word order is not common (although such sentences are completely grammatical), the presentation of such stimuli might have induced the participants to read them more carefully, thus imposing an extra load on working memory, having an effect similar to that of mental rehearsal in general. This conjecture conforms with [16]'s finding that the aLIFG is activated when syntactic information is processed at the sentence level without explicit instructions, and that the pLIFG is additionally activated when explicit syntactic decisions are required. This interpretation of the functional distinction between the aLIFG and pLIFG receives further support from studies on sentence processing in second language learning. For instance, [38] asked advanced learners of Japanese (who were native speakers of Chinese or Korean) to read sentences such as (1) above. Unlike native speakers of Japanese, both the Chinese and Korean learners exhibited enhanced activation in the pLIFG 
rather than the aLIFG in the noncanonical OS condition compared with the canonical SO condition. This could be because the participants, though advanced learners, had less experience of the noncanonical word order, which therefore required conscious processing.

A related discussion can be seen in [39], who report an interesting experimental result concerning the pLIFG. They compared the brain activations of 13-year-old twins, whose native language was Japanese, before and after a certain period of English instruction. The tasks involved two languages: a past-tense-matching task in Japanese and a verbform-matching task (regular and irregular inflection) in English. The twins showed prominent activation in the pLIFG during the English verb form processing task. [35] claimed that since the task of Japanese past tense judgment evoked significant activation in the same brain region, it can be assumed that the twins uniformly handled the stimuli in both Japanese and English. Therefore, they concluded that in both the languages, explicit knowledge (such as the morphological alternation of tense markers) requires young participants to employ an attentive processing strategy. Importantly, the same past-tense-matching task for Japanese evoked activation in the aLIFG for adult native speakers of the language, who can be assumed to be more experienced in inflecting Japanese verbs for tense. Thus, the pLIFG can bear a more conscious recognition of syntactic forms such as AND.

\section{CONCLUSION}

We investigated how S-scrambling and M-scrambling affected brain activation so that we could clarify the relationship among grammatical knowledge, processing components, and neural substrates in sentence processing. S-scrambling enhanced activation in the aLIFG in ditransitive sentences as did M-scrambling in (mono-)transitive sentences, and the latter enhanced activation in the pLIFG in ditransitive sentences. These results are more congruent with the process-based approach to neural bases for sentence processing than with the grammar-based one.

\section{CONFLICT OF INTEREST}

None declared.

\section{ACKNOWLEDGEMENT}

This study was supported by a Grant-in-Aid for Scientific Research (S) (No. 22222001 to M.K.) from the Japan Society for the Promotion of Science.

\section{REFERENCES}

[1] Bahlmann J, Rodriguez-Fornells A, Rotte M, Munte TF. An fMRI study of canonical and noncanonicalword order in German. Hum Brain Mapp 2007; 28: 940-9.

[2] Ben-Shachar M, Palti D, Grodzinsky Y. Neural correlates of syntactic movement: Converging evidence from two fMRI experiments. Neuroimage 2004; 21: 1320-36.

[3] Bornkessel I, Zysset S, Friederici AD, von Cramon DY, Schlesewsky M. Who did what to whom? The neural basis of argument hierarchies during language comprehension. Neuroimage 2005; 26: 221-33.
[4] Bornkessel-Schlesewsky I, Schlesewsky M, von Cramon DY. Word order and Broca's region: evidence for a supra-syntactic perspective. Brain Lang 2009; 111: 125-39.

[5] Caplan D, Waters GS. Verbal working memory and sentence comprehension. Behav Brain Sci 1999; 22: 77-94.

[6] Caplan D, Stanczak L, Waters G. Syntactic and thematic constraint effects on blood oxygenation level dependent signal correlates of comprehension of relative clauses. J Cogn Neurosci 2008; 20: 64356.

[7] Cooke A, Zurif EB, DeVita C, et al. Neural basis for sentence comprehension: Grammatical and short-term memory components. Hum Brain Mapp 2001; 15: 80-94.

[8] Fiebach CJ, Schlesewsky M, Lohmann G, von Cramon DY, Friderici AD. Revisiting the role of Broca's area in sentence processing: syntactic integration versus syntactic working memory. Hum Brain Mapp 2005; 24: 79-91.

[9] Grewe T, Bornkessel I, Zysset S, Wiese R, von Cramon DY, Schlesewsky M. The emergence of the unmarked: a new perspective on the language-specific function of Broca's area. Hum Brain Mapp 2005; 26: 178-90.

[10] Kuperberg GR, McGuire PK, Bullmore ET, et al. Common and distinct neural substrates for pragmatic, semantic, and syntactic processing of spoken sentences: an fMRI study. J Cogn Neurosci 2000; 12: 321-41.

[11] Musso M, Moro A, Glauche V, et al. Broca's area and the language instinct. Nat Neurosci 2003, 6: 774-81.

[12] Novick JM, Trueswell JC, Thompson-Schill SL. Cognitive control and parsing: Reexamining the role of Broca's area in sentence comprehension. Cogn Affect Behav Neurosci 2005; 5: 263-81.

[13] Sakai KL. Language acquisition and brain development. Science 2005; 310: 815-9.

[14] Rogalsky C, Matchin W, Hickok G. Broca's area, sentence comprehension, and working memory: An fMRI study. Front Hum Neurosci 2008: 2, Article 14 (www.frontiersin.org).

[15] Caplan D, Alpert N, Waters G, Olivier A. Activation of Broca's area by syntactic processing under conditions of concurrent articulation. Hum Brain Mapp 2000; 9: 65-71.

[16] Hashimoto R, Sakai KL. Specialization in the left prefrontal cortex for sentence comprehension. Neuron 2002; 35: 589-97.

[17] Hickok G, Poeppel D. The cortical organization of speech processing. Nat Rev Neurosci 2007; 8: 393-402.

[18] Hoji H. Logical form constraints and configurational structures in Japanese. Doctoral Dissertation, University of Washington, 1985.

[19] Kinno R, Kawamura M, Shioda S, Sakai KL. Neural correlates of noncanonical syntactic processing revealed by a picture-sentence matching task. Hum Brain Mapp 2008; 29: 1015-27.

[20] Saur D, Kreher BW, Schnell S, et al. Ventral and dorsal pathways for language. Proc Natl Acad Sci USA 2008; 105: 18035-40.

[21] Kinno R, Kawamura M, Shioda S, Sakai KL. Neural correlates of noncanonical syntactic processing revealed by a picture-sentence matching task. Hum Brain Mapp 2008; 29: 1015-27.

[22] Kim J, Koizumi M, Ikuta N, et al. Scrambling effects on the processing of Japanese sentences: An fMRI study. J Neurolinguistics 2009; 22(1): 151-66.

[23] Koizumi M, Tamaoka K. Psycholinguistic evidence for the VPinternal subject position in Japanese. Linguistic Inq 2010; 41(4): 663-80.

[24] Saito M. Some asymmetries in Japanese and their theoretical implications. Doctoral Dissertation, MIT, 1985.

[25] Tada H. A/A-bar partition in derivation. Doctoral Dissertation, MIT, 1993.

[26] Mahajan A. A/A-bar distinction and movement theory. Doctoral Dissertation, MIT, 1990.

[27] Hoji H. Logical form constraints and configurational structures in Japanese. Doctoral Dissertation, University of Washington, 1985.

[28] Koizumi M, Tamaoka K. Cognitive processing of Japanese sentences with ditransitive verbs. Gengo Kenkyu (J Linguistic Soc Japan) 2004; 125: 173-90.

[29] Miyagawa S, Tsujioka T. Argument structure and ditransitive verbs in Japanese. J East Asian Linguist 2004; 13: 1-38.

[30] Friederici AD, Schlesewsky M, Fiebach C. WH-movement versus scrambling: the brain makes a difference. In: Karimi S, Eds. Word Order and Scrambling. Malden, USA: Blackwell Publishing 2003; 325-44.

[31] Hagiwara H, Soshi T, Ishihara M, Imanaka K. A topographical study on the event-related potential correlates of scrambled word 
order in Japanese complex sentences. J Cogn Neurosci 2007; 19: 175-93.

[32] Koso A, Hagiwara H, Soshi T. What a multi-channel EEG system reveals about the processing of Japanese double object constructions. IEIC Technical Report. Institute of Electronics, Information and Communication Engineers 2004; 104: 31-6.

[33] Rösler F, Pechmann T, Streb J, Röder B, Hennighausen E. Parsing of sentences in a language with varying word order: Word-by-word variations of processing demands are revealed by event-related brain potentials. J Mem Lang 1998; 38: 150-76.

[34] Ueno M, Kluender R. Event-related brain indices of Japanese scrambling. Brain Lang 2003; 86: 243-71.

[35] Grodzinsky Y, Friederici AD. Neuroimaging of syntax and syntactic processing. Cur Opin Neurobiol 2006; 16: 240-6.
[36] Oldfield R. The assessment and analysis of handedness: the Edinburgh inventory. Neuropsychologia 1971; 9: 812-5.

[37] Friederici AD, Fiebach CJ, Schlesewsky M, Bornkessel ID, von Cramon DY. Processing linguistic complexity and grammaticality in the left frontal cortex. Cereb Cortex 2006; 16: 1709-17.

[38] Kim J, Koizumi M, Yokoyama S, et al. The left inferior frontal gyrus subserves the same function in first and second language acquisition: an fMRI study of Japanese transitive verbs. Poster presented at the 12th annual meeting of Hum Brain Mapp 2006; June 11-15; Florence, Italy.

[39] Sakai KL, Miura K, Narafu N, Muraishi Y. Correlated functional changes of the prefrontal cortex in twins induced by classroom education of second language. Cereb Cortex 2004; 14(11): 1233-9.

Received: February 23, 2011

(C) Koizumi et al.; Licensee Bentham Open.

This is an open access article licensed under the terms of the Creative Commons Attribution Non-Commercial License (http://creativecommons.org/ licenses/by-nc/3.0/), which permits unrestricted, non-commercial use, distribution and reproduction in any medium, provided the work is properly cited. 